\title{
Dispositif d'apprentissage collaboratif en ligne pour développer la compréhension et la production des expressions idiomatiques en FLE auprès des étudiants de la faculté de pédagogie de Minia
}

\section{Mohamed Gomaa Refaï Ibrahim}

Maître de conférences en didactique du FLE Faculté de pédagogie - Université de Minia

\section{Résumé :}

Les expressions idiomatiques sont grandement utilisées dans les communications quotidiennes des Français; les apprenants du français langue étrangère ont besoin d'apprendre ces expressions pour que leur communication se caractérise par la naturalité et la spontanéité. La recherche actuelle vise à développer la compréhension et la production des expressions idiomatiques en français langue étrangère auprès les étudiants de la $4{ }^{\text {ème }}$ année de la faculté de pédagogie de Minia. Pour atteindre cet objectif, le chercheur a élaboré un dispositif d'apprentissage collaboratif en ligne dont les activités sont synchrones et asynchrones (lecture collaborative, écriture collaborative, recherche en ligne, visioconférence). Les outils et les applications utilisés dans ce dispositif sont Blog, Google Classroom, Google docs, Google forms, Calendrier Google, Google Meet, Gmail. Pour mesurer l'efficacité du dispositif proposé, le chercheur a élaboré un pré-post/test sur la compréhension et la production des expressions idiomatiques. Notre expérimentation a eu lieu pendant le premier semestre de l'année universitaire 2020/2021, un échantillon de 57 étudiants ont participé à l'expérimentation; cet échantillon a été réparti en deux groupes un groupe expérimental (30 étudiants) et un groupe témoin (27 étudiants). Les résultats de l'expérimentation ont démontré l'efficacité du dispositif proposé de l'apprentissage collaboratif en ligne pour développer la compréhension et la production des expressions idiomatiques. Cette recherche recommande d'utiliser l'apprentissage collaboratif en ligne pour développer la compréhension et la production des expressions idiomatiques.

Mots-clés : Dispositif, Apprentissage collaboratif, Expressions idiomatiques, phraséologie, compétences sociolinguistiques 


\section{بيئة تعلم تشاركي عبر الانتزنت لتنسية فهم وإنتاج التعبيرات الاصطلاحية بالاغة الفرنسية كلغة أجنبية لدى طلاب كلية التربية جاهعة المنيا} محمل جمعة رفاعي إبراهيم

مدرس المناهج وطرق تدريس اللفة الفرنسية

\section{كلية التربية- -جامعة المنيا}

مستخلص :

تستخدم التعبير ات الاصطلاحية على نطاق و اسع في الاتصالات اليومية للفرنسبين؛ ويحتاج متعلمو اللغة الفرنسية كلغة أجنبية إلى تعلم هذه التعبيرات حتى يتسم تو اصلهم بالطبيعة و العفوية. يهدف البحث الحالي إلى تتمية فهم و إنتاج التعبيرات الاصطلاحية باللغة الفرنسية كلغة أجنبية لدى طلاب الفرقة الر ابعة بكلية التزبية جامعة المنيا. ولتحقيق هذا الهدف، أعد الباحث بيئة تعلم تشاركي عبر الإنترنت تعتمد أنشطته على القزاعة

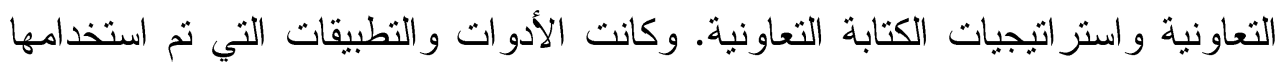

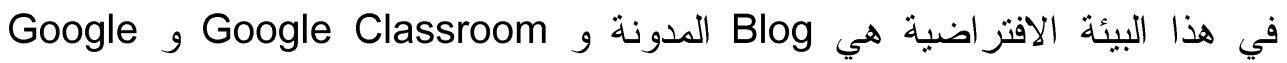

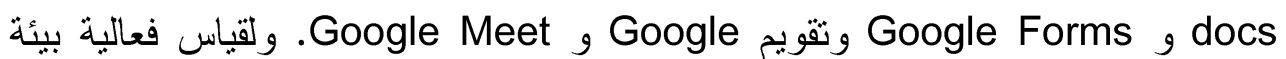
التعلم المقترحة ، أعد الباحث اختبارًا قبلباً/بعدياً عن فهم و إنتاج التعبير ات الاصطلاحية باللغة الفرنسية كلغة أجنبية. تمت التجربة خلال الفصل الدراسي الأول من العام الجامعي 2020/2021 ، وشارك في التجربة OV طالبًا وطالبة. ثم تقسيم هذه العينة إلى الى مجمو عتين مجموعة تجريبية ( •r طالباً وطالبةً) ومجمو عة ضابطة (rV طالباً وطالبةً). أظهرت نتائج التجربة فاعلية بيئة التعلم التشاركي عبر الإنترنت المقترح في تتمية فهر و إنتاج التعبيرات الاصطلاحية. ويوصي هذا البحث باستخدام التعلم التشاركي عبر الإنري الإنترنت لتتمية فه و إنتاج التعبير ات الاصطلاحية باللغة الفرنسية كلغة أجنبية. كلمات مفتاحية : بيئة تعلم افتر اضية ، التعلم التشاركي ، التعبيرات الاصطلاحية، علم العبار ات، المهار ات اللغوية الاجتماعية 


\section{Introduction :}

La langue n'est pas un simple système vocalique ou graphique qu'on utilise sans avoir recours à d'autres composantes essentielles dans la communication surtout les composantes culturelles. De même, La langue doit être considérée comme un miroir qui reflète une culture spécifique. Cette culture forme l'identité des individus d'une nation et de leur langue. Pour comprendre des interlocuteurs natifs et leur faire comprendre, on doit avoir conscience, à côté de la langue qu'ils parlent, de leur culture afin qu'ils comprennent le sens exact du message adressé ; ce message soit codé et décodé de la même manière.

La communication en langue étrangère est un processus de compréhension de l'autre qui est d'une culture différente, le problème réside dans l'intercompréhension pour ces deux interlocuteurs dans l'échange de leurs idées. Dans cette communication, le contexte culturel joue un rôle très important. Or ,la communication interculturelle est à la rencontre de deux cultures différentes. Si le message adressé est codé selon le contexte culturel de l'émetteur et décodé d'une manière différente selon un autre contexte culturel propre au destinataire, la communication devient difficile et peut être source d'incompréhension. Si le locuteur ne possède pas une connaissance suffisante de la langue et de la culture étrangères, il mélange la culture maternelle et la culture étrangère, ce qui aboutira à des malentendus (Zhang, 2012).

Le compétences communicatives langagières peuvent être décomposées en des compétences linguistiques : lexique, grammaire, sémantique (organisation du sens), phonologie (phonétique et prosodie), orthographie, orthoépie (correspondance phonie-graphie), des compétences pragmatiques :compétences discursive (organisation des phrases) et fonctionnelle (actes de parole) et de la compétence sociolinguistique qui correspond au fonctionnement de la langue des points de vue sociaux : marqueurs des relations sociales, règles de politesse, expressions de la sagesse populaire, différences de registre, dialectes et accents (Teixeira, 2011).

La compétence sociolinguistique fait partie intégrante de la compétence communicative en ce qu'elle comprend l'apprentissage 
des connaissances pragmatiques et sociolinguistiques sur la manière d'utiliser la langue linguistiquement et socialement appropriée (Mede et Dikilitaş, 2015). La compétence sociolinguistique est décrite comme l'habileté à reconnaître les variétés de la langue et à utiliser les formes linguistiques appropriées en fonction de la situation de communication (Nussbaum et Unamuno, 2006).

Le Cadre Européen Commun de Référence (Cadre, 2001, p.18) a déclaré que la compétence sociolinguistique renvoie aux paramètres socioculturels de l'utilisation de la langue. Cette compétence est sensible aux normes sociales (les règles de politesse et d'adresse, la régulation des rapports entre les générations, les sexes, les groupes sociaux, ...). Or, la compétence sociolinguistique affecte toutes les communications langagières entre les représentations de cultures différentes.

De même, la compétence sociolinguistique nécessite d'ajuster ses formes grammaticales pour qu'elles soient adaptées au contexte dans lequel se déroule la communication. Une attention est accordée à des facteurs tels que l'âge, le statut, le sexe des participants et la formalité du lieu. Par conséquent, pour apprendre à bien parler dans la culture de la langue cible, il est nécessaire que les étudiants étudient la culture et les différences interculturelles pour pouvoir déterminer en quoi leur culture d'origine diffère de la culture de la langue visée (Mizne, 1997).

Plusieurs études ont souligné le manque des compétences sociolinguistiques en communication auprès les apprenants, quel que soit leur niveau de compétence linguistique. Mizne (1997) a affirmé que les apprenants d'une langue étrangère, même après avoir étudié cette langue pendant de nombreuses années, éprouvent de grandes difficultés à s'exprimer dans cette langue auprès d'un locuteur natif. L'apprenant d'une langue peut prononcer un énoncé parfaitement grammatical que l'auditeur natif peut comprendre, mais le locuteur ne peut pas connaître le sens social voulu. Les raisons de cette difficulté persistante à communiquer dans la langue étrangère peuvent comporter des problèmes de prononciation, un manque de connaissances sur l'utilisation réelle de la parole d'expressions idiomatiques et d'argot. Un autre facteur important contribuant à 
l'incompétence dans la langue est que le locuteur ne sait pas quels énoncés sont appropriés dans la situation sociale dans laquelle il parle.

L'un des facteurs qui rend la compétence sociolinguistique si difficile à acquérir est la grande variance des règles culturelles de la parole; en d'autres termes, ce qui est approprié de dire dans une culture peut être totalement inappropriée dans une autre culture, même si la situation de communication est identique. L'apprenant ignore souvent ces différences et utilise les règles pour parler de sa culture d'origine lorsqu'il communique dans une langue étrangère. $\mathrm{Ce}$ processus, appelé transfert pragmatique, entraîne des malentendus entre les interlocuteurs et peut entraîner de graves problèmes de communication (Mizne, 1997).

Les apprenants d'une langue étrangère trouvent, souvent, des difficultés dans la compréhension de la langue surtout quand ils communiquent avec des interlocuteurs natifs et quand ils écoutent des enregistrements, des émissions, des films préparés par les natifs ; c'est parce que ceux-ci utilisent des expressions qu'on ne peut pas les traduire mot à mot, mais elles ont des sens figés, c'est ce qu'on appelle les expressions idiomatiques.

Alwadi et Alhathal (2013) affirment que les difficultés observées auprès les apprenants dans l'apprentissage de la langue française ne résident pas souvent dans une méconnaissance de la langue (sa phonologie, sa sémantique ou sa syntaxe). Mais, elles résident, en particulier, dans l'ignorance $d u$ référent culturel français. L'enseignement de la culture française, à côté de l'enseignement du français, devient un impératif pédagogique. Il ne suffit plus d'enseigner le français, mais, il s'agit de communiquer en français. Par conséquent, il est nécessaire d'enseigner cette langue en prenant en considération trois dimensions : linguistique, communicative et culturelle.

Les expressions idiomatiques constituent des difficultés de compréhension et de traduction puisqu'elles possèdent des structures syntaxiques particulières qui n'ont pas, souvent, de correspondants analogues dans la langue maternelle. Ces expressions représentent des structures imprévisibles à l'égard de la sémantique en raison du 
manque de correspondance entre le sens de chacun des éléments constitutifs et le sens global de l'expression. L'usager qui ne connaît pas l'expression idiomatique essaie de trouver le sens à partir de la traduction littérale de l'expression. Cette traduction littérale peut modifier le sens de l'expression ou mener à un sens non plausible (Da Silva et Ponge, 2012).

Récemment, les études dans le domaine de la didactique des langues s'intéressent à l'enseignement des expressions idiomatiques. L'étude de Laval \& Bernicot (2002) a visé à déterminer le rôle des caractéristiques contextuelles et de la convention linguistique dans la compréhension des expressions idiomatiques auprès les enfants de 6 et 9 ans et auprès les adultes. La tâche du sujet était de terminer des histoires. Douze histoires ont été présentées sous forme de bande dessinée par l'expérimentateur, qui a raconté l'histoire dans les trois premiers cadres et a ensuite demandé au sujet de choisir l'une des deux fins possibles. Deux caractéristiques des histoires étaient variées : le contexte de production de l'énoncé (idiomatique - littéral) et le niveau de familiarité idiomatique (familier - non familier). Quel que soit l'âge, le contexte a eu un impact substantiel sur la compréhension des idiomes : cela renforce l'idée de la nécessité de prendre en compte le contexte et les conventions extralinguistiques pour expliquer le fonctionnement du langage, auprès l'enfant et l'adulte.

L'étude de Pulido, Iralde et Weil-Barais (2010) a porté sur l'émergence de la compréhension des expressions idiomatiques auprès les élèves de maternelle. Les auteurs ont présenté dix séances de lecture des contes contenant des expressions idiomatiques différenciées selon des critères de familiarité, de transparence métaphorique et de plausibilité littérale. Les résultats ont montré que les élèves sont capables d'accéder au sens de certaines de ces expressions en s'appuyant notamment sur les éléments contextuels.

L'étude de Mede et Dikilitaş (2015) a visé à explorer les perceptions des apprenants ainsi que les connaissances des enseignants d'anglais langue étrangère non natifs sur les compétences sociolinguistiques ainsi que toute difficulté qu'ils peuvent rencontrer avec l'intégration de cette compétence particulière dans leur pratique en classe. Les résultats de cette étude ont révélé que l'élaboration de 
règles sociolinguistiques peut guider les apprenants dans le choix des formes appropriées qui devraient être étroitement intégrées dans les programmes d'enseignement et d'apprentissage des langues.

L'étude de Khoshhal et Hassasskhah (2017) a visé à déterminer comment les apprenants en anglais choisissent leurs stratégies préférées pour trouver la signification correcte des phrases idiomatiques dans un texte. Trente-trois participants étaient répartis en deux groupes. Le groupe expérimental (avec dix-sept étudiants) a appris explicitement les idiomes pendant le cours et le groupe témoin (avec seize étudiants) n'a reçu aucune instruction explicite dans l'apprentissage des idiomes. Les résultats ont montré que parmi les stratégies données, le groupe qui a été enseigné avait explicitement tendance à choisir «d'utiliser le dictionnaire» plus souvent tandis que l'autre groupe préférait «demander à l'enseignant». Les participants utilisent de différentes stratégies et techniques lorsqu'ils rencontrent un problème, mais chaque élève a une façon particulière d'apprendre. Cette étude suggère que l'enseignement explicite des idiomes devrait être combiné avec l'apprentissage implicite dans les contextes. En outre, les apprenants ont besoin d'une plus grande exposition (explicite ou implicite) à la signification et à l'utilisation des phrases idiomatiques afin de maîtriser ce domaine d'apprentissage complexe.

Pour développer la compétence sociolinguistique, les étudiants peuvent apprendre explicitement ou implicitement des éléments culturels ancrés dans l'utilisation de la langue et intègrent des actes de langage en tant que situations où les apprenants sont obligés d'utiliser la langue en tenant compte de facteurs socio-pragmatiques tels que les statues sociales de l'auditeur. Puisque la concentration principale de la plupart des apprenants est consacrée à apprendre quelles sont les règles de la langue et comment ils peuvent produire des phrases grammaticales plutôt que comment ils peuvent produire des phrases appropriées qui correspondent au contexte social spécifique (Mede et Dikilitaş, 2015).

L'étude de Lyster (1994) a examiné l'effet de l'enseignement analytique fonctionnel sur certains aspects de la compétence sociolinguistique (définie comme la capacité de reconnaître et de produire une langue socialement appropriée dans le contexte) des 
élèves inscrits dans un programme d'immersion française en $8 \mathrm{e}$ année. L'étude a été mise en œuvre dans trois salles de classe sur une période moyenne de cinq semaines. Cette étude a démontré que les étudiants qui ont suivi un enseignement analytique fonctionnel ont développé considérablement les aspects de la compétence sociolinguistique (en comparaison avec le groupe contrôle qui a suivi un programme régulier basé sur une approche expérientielle ne visant aucun aspect sociolinguistique de manière intentionnelle) de trois façons: (I) en augmentant considérablement leur capacité en production orale à utiliser de manière appropriée et précise vous dans des situations formelles; (2) en augmentant considérablement leur capacité dans la production écrite à utiliser correctement vous dans les lettres officielles et, à court terme, à utiliser des clôtures polies dans les lettres formelles; et (3) en augmentant considérablement leur capacité à reconnaître le français approprié au contexte.

L'objectif de l'étude de Ritchie (2011) était de déterminer si l'échange interculturel de communication assisté par ordinateur offre les conditions nécessaires au développement de la compétence sociolinguistique des apprenants de langue seconde. Des locuteurs non natifs du français en Colombie-Britannique ont interagi par l'entremise de communication assistée par ordinateur avec des locuteurs natifs du français au Québec au cours d'un semestre universitaire. S'appuyant sur la perspective socioculturelle, cette étude a utilisé une approche qualitative pour analyser les données collectées. Les données comprenaient les transcriptions des discussions de clavardage textuel et d'un forum de discussion. Les résultats de cette étude suggèrent que l'échange interculturel de communication assisté par ordinateur offre des conditions positives pour le développement de la compétence sociolinguistique. Les résultats permettent également une description générale des éléments sociolinguistiques impliqués dans ce type d'échange.

Les études antérieures ont montré que la pédagogie basée sur la collaboration aide à accroître la tolérance, le respect des autres et l'estime de soi. Elle contribue, également, à améliorer la pensée critique, les capacités de résoudre des problèmes, de créer une dynamique ascendante permettant une construction étape par étape 
des connaissances (Johnson, 1994 ; Qin, Johnson et Johnson, 1995, Palloff \& Pratt, 2005, Stoytcheva, 2018).

L'apprentissage collaboratif est une méthode intéressante à explorer pour générer des conditions pédagogiques favorables pour développer les attitudes de tolérance et d'ouverture aux différences culturelles. C'est une notion qui inclut des méthodes pédagogiques qui appliquent des activités de coopération et des travaux réalisés en équipe, comme dans l'apprentissage par problèmes et l'apprentissage par projet réalisé. Cette méthode permet de créer des scénarios proches de la réalité où les étudiants ont à négocier au sujet de leur manière de faire ou de réagir dans la réalisation de la tâche proposée. Le terme collaboration fait référence à toute activité où les étudiants sont invités à exécuter des tâches ou des problèmes d'une manière collective, chacun des membres de l'équipe est responsable de ramener à l'équipe à un résultat réussi de ses travaux. Tous les membres de l'équipe doivent maîtriser le contenu de la matière à l'étude et répondre aux exigences de l'enseignant, tout en devant se faire confiance les uns aux autres et négocier constamment entre eux. Collaborer pour apprendre s'inscrit parmi les théories socioconstructivistes; cette méthode permet aux étudiants de construire collectivement un savoir en les invitant à mettre en commun leurs connaissances. Par conséquent, le rôle de l'enseignant se modifie ; puisque l'enseignant n'est plus l'unique expert et son rôle n'est plus celui d'un «transmetteur» de connaissances. Mais, il doit veiller à la qualité des renseignements acquis et à les compléter au besoin. De même, l'apprentissage collaboratif favorise le recours au feedback des uns envers les autres puisque les étudiants doivent interagir pour exécuter les tâches demandées (Denis, 2005).

\section{Problématique de la recherche :}

Cavalla et Labre (2009) déclarent que les réflexions et les constats d'enseignants étrangers de français permettent de mettre en évidence certaines difficultés d'apprentissage face aux expressions idiomatiques. Des enseignants de FLE non-natifs avouent avoir du mal à retenir ces expressions car ils ne savent pas toujours à quelles situations les associer exactement et préfèrent, dans ce cas, ne pas les utiliser. 
Tamayo (2017) a montré que les expressions idiomatiques ont toujours été peu étudiées en classe de FLE car elles ne sont pas, dans la plupart des cas, traitées dans les manuels de FLE. Les étrangers, voyageurs en France, remarquent que les expressions idiomatiques sont très utilisées par les Français. Des expressions comme poser un lapin, mettre une mine, donner sa langue au chat, ou avoir la grosse tête, sont très utilisées dans la rue. L'étude des expressions françaises est indispensable pour les étudiants de FLE, et c'est grâce à une nouvelle discipline, la phrasé didactique, que les expressions figées trouvent leur place dans la didactique de la langue française.

Les étudiants en langues étrangères, en particulier les étudiants de français langue étrangère, n'ont pas la capacité d'utiliser la langue étrangère automatiquement et perdent naturellement et automatiquement des compétences de parole qui simulent la façon dont les locuteurs natifs parlent. Cela est souvent dû au fait que la langue n'a pas seulement besoin d'apprendre la grammaire et le vocabulaire, mais a besoin d'apprendre des expressions idiomatiques afin que l'utilisation naturelle et automatique de la langue imite la langue parlée par des locuteurs natifs.

Le chercheur a observé pendant l'enseignement aux étudiants de la faculté de pédagogie, université de Minia que les étudiants de $4^{\text {ème }}$ année (en tant que des étudiants dans la dernière année de la formation des enseignants du FLE) ne peuvent pas comprendre les expressions idiomatiques dans les conversations en français. Ils ont recours, souvent, à la traduction littérale des expressions idiomatiques du français. De même, ils ne peuvent pas utiliser les structures et les expressions idiomatiques du français dans des situations adéquates. Les expressions idiomatiques sont presque absentes dans leurs conversations et écritures.

Le chercheur a fait une étude exploratoire pour s'assurer de ce problème présenté. Un test a été administré auprès des étudiants de la $4^{\mathrm{e}}$ année de la faculté de pédagogie de Minia (test administré en ligne sur le site français facile). Les résultats du test ont montré que les étudiants de la $4^{\text {ème }}$ année de la faculté de pédagogie de Minia ont de grandes difficultés dans la compréhension et la production des 
expressions idiomatiques. Pour envisager ce problème, la recherche actuelle va répondre aux questions suivantes :

1. Comment élaborer un dispositif proposé d'apprentissage collaboratif en ligne pour développer la compréhension et la production des expressions idiomatiques en français langue étrangère auprès les étudiants de la $4^{\mathrm{e}}$ année de la faculté de pédagogie de Minia?

2. Quelle est l'efficacité du dispositif proposé d'apprentissage collaboratif en ligne pour développer la compréhension des expressions idiomatiques en français langue étrangère auprès les étudiants de la $4^{\mathrm{e}}$ année de la faculté de pédagogie de Minia ?

3. Quelle est l'efficacité du dispositif proposé d'apprentissage collaboratif en ligne pour développer la production des expressions idiomatiques en français langue étrangère auprès les étudiants de la $4^{\mathrm{e}}$ année de la faculté de pédagogie de Minia ?

\section{Objectifs de la recherche :}

1. Développer la compréhension des expressions idiomatiques en français langue étrangère auprès les étudiants de la $4^{\mathrm{e}}$ année de la faculté de pédagogie de Minia ;

2. Développer la production des expressions idiomatiques en français langue étrangère auprès les étudiants de la $4^{\mathrm{e}}$ année de la faculté de pédagogie de Minia ;

3. Vérifier l'efficacité d'un dispositif proposé d'apprentissage collaboratif en ligne pour développer la compréhension des expressions idiomatiques en français langue étrangère auprès les étudiants de la $4^{\mathrm{e}}$ année de la faculté de pédagogie de Minia ;

4. Vérifier l'efficacité d'un dispositif proposé d'apprentissage collaboratif en ligne pour développer la production des expressions idiomatiques en français langue étrangère auprès les étudiants de la $4^{\mathrm{e}}$ année de la faculté de pédagogie de Minia ; 


\section{Délimitation de la recherche :}

Cette recherche se délimite :

1- Aux expressions idiomatiques les plus familières et les plus utilisées en français.

2- A quelques outils et applications de la collaboration en ligne les plus adéquats pour les objectifs de la recherche.

3- Aux étudiants de la $4^{\text {ème }}$ année de la faculté de pédagogie de Minia parce qu'ils possèdent un bagage linguistique et leur niveau langagier leur permet d'apprendre les expressions idiomatiques.

\section{Hypothèse de la recherche :}

1. Il y a une différence statistiquement significative entre la moyenne des notes des étudiants du groupe expérimental au pré-post test concernant la compréhension des expressions idiomatiques en français langue étrangère en faveur du posttest.

2. Il y a une différence statistiquement significative entre la moyenne des notes des étudiants du groupe expérimental au pré-test et celle au post-test concernant la production des expressions idiomatiques en français langue étrangère en faveur du post-test.

3. Il y a une différence statistiquement significative entre la moyenne des notes des étudiants du groupe expérimental et celle du groupe témoin au post-test concernant la compréhension des expressions idiomatiques en français langue étrangère en faveur du groupe expérimental.

4. Il y a une différence statistiquement significative entre la moyenne des notes des étudiants du groupe expérimental et celle du groupe témoin au post-test concernant la production des expressions idiomatiques en français langue étrangère en faveur du groupe expérimental.

\section{Outils et matériels de la recherche :}

1- Un pré-post test en vue de mesurer l'efficacité du dispositif proposé d'apprentissage collaboratif en ligne pour développer la compréhension et la production des expressions idiomatiques en français langue étrangère auprès les étudiants de la $4^{\mathrm{e}}$ année de la 
faculté de pédagogie de Minia (ce test est divisé en deux parties : compréhension des expressions idiomatiques et production des expressions idiomatiques);

2- Un dispositif proposé d'apprentissage collaboratif en ligne pour développer la compréhension et la production des expressions idiomatiques en français langue étrangère auprès les étudiants de la $4^{\mathrm{e}}$ année de la faculté de pédagogie de Minia.

\section{Echantillon de la recherche :}

Cette recherche se délimite à un échantillon aléatoire (57 étudiants) parmi étudiants de la $4^{\text {ème }}$ année de la faculté de pédagogie de Minia (46 étudiantes et 11 étudiants) parce qu'ils possèdent un bagage linguistique et leur niveau langagier leur permet d'apprendre les expressions idiomatiques. Cet échantillon est distribué en deux groupes : un groupe expérimental (30 étudiants) et un groupe témoin (27 étudiants).

\section{Méthodologie de la recherche :}

Afin de réaliser cette recherche, le chercheur utilise une méthode descriptive pour présenter le cadre théorique. Pour vérifier l'efficacité $\mathrm{du}$ dispositif proposé d'apprentissage collaboratif en ligne pour développer la compréhension et la production des expressions idiomatiques en français langue étrangère auprès les étudiants de la $4^{\mathrm{e}}$ année de la faculté de pédagogie de Minia, le chercheur utilise la méthode quasi-expérimentale en utilisant un groupe expérimental et un groupe témoin avec une pré/post évaluation.

\section{Terminologie de l'étude :}

\section{Dispositif :}

Holtzer (2002 : 14) définit le dispositif comme « un ensemble organisé de structures, de ressources et de moyens mis en place en fonction d'un projet pédagogique. Un dispositif de formation ... combine des espaces d'apprentissage pluriels (salles de cours, centre de ressources, espaces de conversation...), chaque espace donnant lieu à des modalités d'apprentissage différentes (apprentissage collectif, en petits groupes, en binôme, individuel) et ayant une 
fonction pédagogique spécifique (enseignement, auto-apprentissage, apprentissage collaboratif, tutorat...».

Dans cette recherche, le dispositif est un groupe d'outils et d'applications du web qui permet le travail en ligne et qui combine les ressources nécessaires pour accéder aux informations et les former pour atteindre des objectifs pédagogiques. (Définition opérationnelle)

\section{L'apprentissage collaboratif en ligne.}

L'apprentissage collaboratif en ligne est une stratégie d'apprentissage qui se base sur les activités synchrones ou asynchrones d'apprentissage en ligne dans lesquelles les apprenants se divisent en groupes dont chaque membre est responsable de son apprentissage et de l'apprentissage de ses pairs afin d'atteindre des objectifs déterminés. (Définition opérationnelle)

\section{Expressions idiomatiques}

Dans le dictionnaire le Petit robert (1991, p. 95) l'expression idiomatique est une «Forme ou locution propre à une langue, impossible à traduire littéralement dans une autre langue de structure analogue ».

De même, dans le Dictionnaire de linguistique Larousse, Dubois (1993, P. 239) déclare qu'" On appelle expression idiomatique toute forme grammaticale dont le sens ne peut être déduit de sa structure en morphèmes et qui n'entre pas dans la constitution d'une forme plus large $\gg$.

Dans cette recherche, les expressions idiomatiques sont des locutions, à connotation culturelle, qui ont des sens figés et qui ne peuvent pas, souvent, être traduites mot à mot. (Définition opérationnelle)

\section{Cadre conceptuelle :}

La langue n'est pas seulement un système de communication pour comprendre et faire comprendre les informations transmises, mais, elle est également un résultat d'un système socioculturel qui inclut une culture, une histoire, des attitudes, des habitudes, des mœurs et des croyances d'un peuple et d'une nation. Par conséquent, pour bien 
apprendre une langue et communiquer efficacement avec les natifs de cette langue, il ne suffit pas d'apprendre le système vocalique, de connaitre les règles de grammaire, d'apprendre par cœur son lexique ; mais, il faut également apprendre le système phraséologique de cette langue.

La compétence phraséologique est une partie complexe de la compétence linguistique et communicative générale en langue parlée et dans d'autres langues maîtrisées. Cela suppose que les locuteurs possèdent une compétence linguistique générale qui comprend l'ensemble des compétences linguistiques, c'est-à-dire des connaissances phonétiques, morphosyntaxiques, grammaticales, lexico-sémantiques, textuelles, situationnelles, pragmatiques et culturelles et la compétence communicative dans une langue donnée. La compétence communicative est ainsi considérée comme la capacité d'utiliser correctement le langage dans la communication. Pour la compétence phraséologique, elle fait partie d'une compétence générale de communication, culturelle et linguistique qui permet à un locuteur de communiquer avec succès dans un contexte socioculturel donné. Cette compétence comprend tout ce qu'une personne doit savoir pour pouvoir comprendre et utiliser correctement la phraséologie dans la communication (Hallsteinsdóttir, In print « sous presse »).

Dans le dictionnaire Larousse, la phraséologie est définie comme le domaine qui concerne la «construction de phrase ou procédé d'expression propre à une langue, à une époque, à une discipline, à un milieu, à un auteur ». Dans le même dictionnaire, la phraséologie est aussi définie comme le «Recueil de phrases ou de locutions caractéristiques d'une langue donnée et destiné à l'enseignement de cette langue ».

La phraséologie est un terme qui désigne l'analyse d'expressions figées d'une langue. Elle est considérée, au sein de la linguistique, comme une discipline autonome qui englobe les caractéristiques des séquences lexicales perçues comme préconstruites et souvent utilisées dans la vie courante. L'originalité de ce type de langage, qu'on retrouve à l'oral et à l'écrit, repose sur la présence d'éléments poly lexicaux, c'est-à-dire, de séquences qui sont composées de plusieurs 
mots, qui à l'origine étaient autonomes, tout en formant des structures unitaires, ce qui permet de les interpréter dans leur ensemble (Gonzalez, 2016).

Pour un manque d'étude dans le domaine de la didactique de la phraséologie, elle constitue actuellement une sorte de lacune placée à mi-chemin entre la phraséologie pure et la didactique des langues (Sułkowska, 2013). La phrasé didactique, qui est devenu présente dans la littérature principalement grâce aux travaux d'auteurs allemands, examine les processus associés à l'assimilation naturelle des idiomes, des proverbes et d'autres formes de mots reproductibles dans la langue. Les expressions idiomatiques sont comprises comme des combinaisons établies d'au moins deux mots avec un caractère reproductible. La portée de la phraséologie comprend les mots composés et les collocations fixes (Sułkowska, 2016).

L'objectif fondamental de la phrasé didactique est la didactique de la phraséologie dans un sens large, c'est-à-dire l'enseignement/apprentissage de tout élément considéré comme unité phraséologique, à savoir les expressions idiomatiques et les collocations. L'acquisition de ces expressions figées doit autant se faire en langue maternelle qu'en langue étrangère dans une approche actionnelle comprenant tous les aspects de la compétence communicative (linguistique, sociolinguistique et pragmatique) (Sułkowska, 2016).

Pour désigner les expressions d'usages de la langue, les auteurs utilisent certains termes tels que les collocations, les locutions, les locutions figées, les expressions figées, les stéréotypes ou les expressions idiomatiques.

Le terme idiomatique vient du lexique « idio» qui veut dire langue «propre à une langue ». Par exemple, en français on dit mettre quelqu'un en boîte pour se moquer de quelqu'un. Ce n'est qu'en français que l'on comprend cette expression. En d'autres langues, on ne pourrait pas comprendre cette expression qui, du point de vue sémantique, serait incompréhensible. Ces expressions idiomatiques sont aussi appelées «expressions imagées», car elles vont nous faire penser à une image (Tamayo, 2017). 
Les expressions idiomatiques représentent un des aspects de la culture de la langue puisqu'elles se forment à travers la vision des choses, de l'environnement et des objets par le peuple dont on parle cette langue. Les expressions idiomatiques ont leurs origines historiques et culturelles qui forment l'identité culturelle de la nation car ces expressions se forment à travers les siècles successifs ; ainsi, elles portent des significations figées et parfois des aspects humoristiques.

La langue et la culture sont deux systèmes sémiotiques en corrélation continue qui s'observent au niveau de la phraséologie car les unités de ce niveau contiennent dans leur forme intérieure un système d'images. Ces images reflètent leurs visions stéréotypées inhérentes à la communauté linguistique donnée. La composante culturelle des idiomes est la composante principale du sens phraséologique qui permet aux unités de la phraséologie de véhiculer à travers leur forme les informations se référant à la culture du peuple (le système de valeurs, les traditions, les symboles, les idéologies). Ainsi, le système phraséologique est un miroir dans lequel se reflète l'esprit du peuple, sa spécificité et sa créativité (Papcov, 2012).

La différence de la culture dans la communication entre les locuteurs natifs et les locuteurs non natifs peut dévoiler des problèmes. Un des problèmes les plus sérieux est celui de la non compréhension et de l'usage insuffisant de la langue dominante. Les locuteurs non natifs peuvent transmettre les messages à travers la langue et non à travers la culture. Les difficultés liées à l'utilisation de la langue ont des conséquences sur la communication, puisque le message ne peut pas être décodé et compris par le récepteur (Nikolaou, 2009).

Par conséquent, les expressions idiomatiques à connotation culturelle sont difficiles à comprendre pour les non natifs d'une langue car leur compréhension exige la mise en œuvre d'un savoir extralinguistique sur la nation dont on parle la langue. La difficulté principale de la communication entre les gens ne vient souvent pas de différences au niveau des systèmes linguistiques, mais des divergences au niveau des expériences des sujets parlants à l'égard de 
l'histoire, de la civilisation, de la tradition littéraire, des stéréotypes sociaux, etc. Pour s'exprimer et comprendre en langue étrangère, il ne suffit pas de connaître les mots, mais il faut aussi maîtriser la signification des groupes de mots qui ont une forme figée et une signification figurée. (Sioridze et Surguladze, 2017).

Pour les locuteurs natifs, ils envisagent quotidiennement les expressions idiomatiques dans leurs conversations. Pour cette raison, ils connaissent bien ce qui veut dire ces expressions, c'est d'une part. D'autre part, les locuteurs natifs envisagent leur propre culture et non une culture étrangère. Pour les apprenants de français du public nonnatif, ils éprouvent souvent des difficultés liées à la compréhension de la signification des expressions idiomatiques ; c'est, souvent, pour deux raisons : les expressions idiomatiques ne font pas partie ni de leurs conversations, ni de leurs situations d'apprentissage puisque les méthodes d'apprentissage de langue ne donnent pas une place suffisante pour l'apprentissage de ces expressions. La deuxième raison, c'est que les interlocuteurs non-natifs envisagent une autre culture qui est tout à fait différente de leur propre culture et que ces expressions peuvent ne pas avoir des analogues dans leur langue maternelle.

Cette complexité est due au registre des expressions idiomatiques qui varie selon le contexte d'énonciation et selon l'interprétation littérale ou figurée de la tournure; ces facteurs constituent une difficulté supplémentaire pour l'apprenant surtout lorsqu'il n'a aucune familiarité avec les expressions idiomatiques. Pour pallier sa méconnaissance idiomatique, l'apprenant en fait des transferts entre la langue maternelle et la langue étrangère. Dans le cas du transfert négatif, ce processus peut représenter un obstacle dans la cohérence et la cohésion discursive, ce qui peut être une rupture dans l'efficacité de la communication (Cáceres-Guerrero, 2017).

Sioridze et Surguladze (2017) mentionnent que l'enseignement des expressions idiomatiques peut poser des difficultés pour les apprenants. Ces difficultés peuvent être liées à la compréhension, à l'emploi ou aux deux : 
- Les difficultés de compréhension: Pour les difficultés de compréhension, les expressions transparentes ne posent pas beaucoup de problèmes (" avoir un cœur d'or »), mais les expressions qui sont plus opaques, empêchent les élèves de connaitre le lien entre l'expression et sa signification. Dans ce cas, l'apprenant d'une langue étrangère peut se tromper s'il traduit l'expression « les carottes sont cuites » mot à mot et il ne comprend pas que cette expression veut dire tout est perdulil n'y a plus rien à faire/il n'y a plus aucun espoir. De plus, il existe des expressions qui portent les traces de la langue ancienne, par exemple, le mot « fur » dans « au fur et à mesure », est un mot vieilli qui signifie « à proportion».

- Les difficultés d'emploi : Quant aux difficultés d'emploi, il ne suffit pas de connaitre seulement l'expression, mais il est important d'être capable de les utiliser dans un bon contexte et d'éviter d'en abuser. Par exemple, l'expression " casser sa pipe » est utilisée seulement pour annoncer la mort de quelqu'un.

Pour Sioridze et Abashidze (2015), les expressions idiomatiques d'une langue, et surtout celles de la langue étrangère, posent beaucoup de problèmes aux étudiants car elles ont les caractéristiques suivantes :

- Les associations de deux ou de plusieurs mots, entièrement figées prennent une nouvelle signification, différente de la signification de ses constituants; ils deviennent comme un tout sémantique à part. II est, alors, impossible de les traduire littéralement.

- Beaucoup d'expressions idiomatiques ont une valeur sémantique ; celle-ci peut être considérée comme spécifique d'une langue. Cette valeur sémantique est souvent empreinte d'une culture et de sa spécificité.

- Les locutions figurées ne peuvent pas être traduites mot à mot puisqu'elles sont chargées d'empreintes culturelles et parfois, elles n'ont pas d'équivalents dans une autre langue. Ces locutions figurées présentent un système de particularités expressives, liées à des conditions sociales dans lesquelles la langue est actualisée. 
Da Silva et Ponge (2012) ajoutent 3 caractéristiques principales pour les expressions idiomatiques : la $1^{\text {ère }}$ caractéristique est qu'elles représentent une unité de forme (groupe syntaxique indécomposable ou forme figée) et de sens (signification qui ne découle pas du sens individuel des termes qui la composent). La deuxième caractéristique porte sur la syntaxe: les expressions idiomatiques sont des locutions qui s'écartent de la norme grammaticale et lexicale. La troisième caractéristique est que la plupart des expressions sont prises dans un sens métaphorique.

Pour la traduction des expressions idiomatiques, il est difficile, dans la plupart des cas de les traduire mot à mot. On peut avoir trois situations différentes dans cette traduction; les expressions idiomatiques peuvent avoir des équivalences totales ; c'est-à-dire les expressions dans les deux langues ont le même lexique et le même sens. Parfois, elles ont des équivalences partielles ; c'est-à-dire, elles ont le même sens, mais avec du lexique différent. Dans certains cas, les expressions idiomatiques n'ont pas d'équivalences, ni au niveau du sens, ni au niveau du lexique. Pour ne pas se tromper dans la compréhension des expressions idiomatiques, il est nécessaire de penser selon la raison de la langue cible et d'avoir recours à la culture.

Il est, alors, nécessaire de présenter aux apprenants d'une langue étrangère un enseignement efficace pour les expressions idiomatiques. De Serres (2011) nous présente treize propositions pédagogiques dans ce domaine ; ce sont les suivantes :

Choix des expressions idiomatiques : Selon cette proposition, on présente les expressions idiomatiques selon trois niveaux : débutant, moyen et avancé. Pour commencer à apprendre les expressions idiomatiques, on peut présenter aux apprenants des séquences brèves avec des mots de haute fréquence et d'une utilisation récurrente à l'oral. Par exemple : chouette ; manger sur le pouce. Pour un niveau mitoyen, on peut présenter des expressions un peu plus longues avec des mots récurrents : avoir du pain sur la planche ; donner sa langue au chat et avoir le feu sacré. Pour un niveau plus élevé : sauter du coq-à-l'âne, vivre aux crochets de quelqu'un et se dilater la rate. 
Stratégies d'enseignement-apprentissage : 1. dire à voix haute l'expression à apprendre ; 2. posséder le sens de l'expression ou l'enseigner ; 3. être familier avec le contexte usuel ; 4. se pencher sur la flexibilité syntaxique d'une expression, par exemple, insérer un adjectif ou un adverbe sans que l'expression ne perde son sens figuré, comme c'est totalement, vraiment, tellement chouette ! 5. se pencher sur la flexibilité lexicale, par exemple, remplacer un mot synonyme un terme de l'expression sans en altérer le sens, comme soit rater soit louper soit manquer le coche.

Associations expression-image-action : On peut associer une expression à des images d'actions concrètes en contexte. L'association des expressions idiomatiques à des images contextuelles peut former une image du sens dans l'esprit de l'apprenant.

Mémorisation : Dans le cas d'une expression opaque, la mémorisation est suggérée. À partir des lectures courantes, l'apprenant relève à l'écrit des expressions dans un carnet personnel et les mémoriser pour en faire part à ses camarades de classe à l'oral.

Résolution de problèmes : Aborder des expressions idiomatiques sous la forme d'une résolution de problèmes est une des méthodes de les apprendre. Mettre l'apprenant au défi de trouver le sens d'expressions opaques (p. ex., prendre la poudre d'escampette) en consultant des locuteurs natifs; de trouver des équivalences dans sa langue maternelle; et de déterminer si les mêmes contextes d'exploitation s'appliquent dans sa langue maternelle et dans la langue étrangère.

Recension, conte et résumé : Trois techniques peuvent être utilisées dans l'enseignement/apprentissage des expressions idiomatiques, c'est recenser les types de textes ou de communications orales où sont présents les expressions idiomatiques. Raconter une histoire déjà lue, et résumer un texte en reprenant les expressions idiomatiques visées constituent une des deux autres techniques pour les assimiler.

Hypothèses et manipulations : La formulation des hypothèses autour des sens des expressions idiomatiques est une des voies de les assimiler et présente un contexte riche de les apprendre. Poser, au 
début de son enseignement, des questions d'anticipation avant de poursuivre avec une manipulation pour favoriser la réflexion et l'apprentissage mobiliserait efficacement l'apprenant. Ces tâches représentent un effort cognitif qui facilite la rétention des expressions en mémoire à long terme.

Regroupements thématiques : Le transfert ou l'approche comparée d'expressions idiomatiques en langue maternelle et en langue étrangère selon des regroupements thématiques représente une des voies de leur rétention et appropriation. Par exemple, les expressions : avoir du pain sur la planche ; casser du sucre sur le dos de quelqu'un ; ménager la chèvre et le chou; se placer en rang d'oignons représente le domaine de la nourriture.

Apprentissage comparatif : La traduction littérale de certaines expressions peut faire une compréhension fautive ; pour cette raison, l'apprentissage comparatif entre la langue maternelle et la langue étrangère peut combler les lacunes de la compréhension.

Proximité sémantique : Aborder les expressions en se basant sur la proximité sémantique pourrait s'avérer une voie d'enseignementapprentissage pertinente à explorer.

Mots-clés : La métaréflexion sur le type de verbe qu'affiche une expression idiomatique, sur sa structure grammaticale sont autant des voies pour parvenir au sens de la forme. Chercher les fondements logico-sémantiques du choix des mots-clés dans une expression idiomatique peut de même représenter un défi attrayant.

Étymologie : Familiariser les apprenants à l'origine étymologique d'une expression idiomatique pourrait être une voie propice pour apprendre les expressions idiomatiques.

Dimension culturelle : La pauvreté de la dimension culturelle peut être la source des difficultés de la compréhension des expressions idiomatiques. Autrement dit, la richesse des situations de l'enseignement/apprentissage des expressions idiomatiques par la dimension culturelle peut faciliter la compréhension des expressions idiomatiques. 
Parmi les méthodes d'enseignement/apprentissage les plus recommandées pendant les dernières années est l'apprentissage collaboratif en ligne. Les outils et les applications de cette méthode permettent aux apprenants de se collaborer pour l'exécution des tâches d'apprentissage dans des situations naturelles semblables à la vie quotidienne. L'apprenant travaille dans un entourage favorable à l'apprentissage ; il devient responsable de ses propres résultats, ainsi que des résultats de ses pairs. L'apprenant devient producteur des connaissances au lieu d'être consommateur des connaissances, il est actif et présente de l'aide à ses pairs.

Les avantages pédagogiques de l'apprentissage collaboratif en ligne comprennent le développement de compétences de pensée critique et de résolution de problèmes ainsi que des compétences d'autoréflexion et de co-construction de connaissances et de sens (Chiong and Jovanovic, 2012).

D'une manière plus explicite, Walckiers et De Praetere (2004) nous présentent huit avantages de l'apprentissage collaboratif en ligne ainsi :

\section{- Flexibilité de temps et autonomie :}

L'apprentissage collaboratif en ligne offre une flexibilité de temps et de lieu. Pour la flexibilité de temps, elle permet à chacun d'effectuer les activités d'apprentissage au moment et au lieu choisi. De même, grâce à cette flexibilité, l'apprenant peut effectuer autrement la même activité et même de faire autre chose. De même, l'apprentissage collaboratif en ligne donne aux apprenants une autonomie qui lui permet de s'interroger, de revenir en arrière, pour réviser les notions déjà étudiées, de reconstruire ses connaissances, de les expérimenter dans leurs activités, de les confronter à celles acquises par leurs pairs, de comparer leurs progrès ou difficultés à ceux de leurs pairs, de trouver par eux-mêmes des informations ou des explications complémentaires sur le web, etc. La flexibilité de l'apprentissage collaboratif en ligne permet aux apprenants des activités variées d'apprentissage adaptées aux besoins et aux intérêts de chacun, elle les amène aussi à gérer leur temps ; c'est l'avantage capital de l'autonomie dans la gestion de l'apprentissage. 


\section{- Délai de réflexion et esprit critique :}

L'avantage majeur de l'apprentissage collaboratif en ligne est le délai de réflexion. Dans l'enseignement présentiel, l'audition de l'exposé d'un enseignant ou d'un pair, ne permet qu'une réflexion limitée, et le cheminement intellectuel suivi s'estompe rapidement dans l'esprit des apprenants. Par contre, dans l'apprentissage collaboratif en ligne, il y a une possibilité accrue de réflexion et d'autonomie d'apprentissage qui favorise une meilleure compréhension et une information complémentaire. Cet avantage pédagogique du délai permet la réflexion de l'apprenant, facilite sa compréhension et stimule son esprit critique.

\section{- La formulation textuelle est plus exigeante et formative :}

L'apprentissage collaboratif en ligne n'exclut pas les communications synchrones : téléphone, visioconférence ou clavardage (chat) ... Mais, la communication textuelle asynchrone (forum et blog) s'impose dans l'apprentissage collaboratif en ligne pour sa flexibilité de temps et de lieu, l'autonomie, le délai de réflexion, la formulation claire et précise... L'autonomie de la communication textuelle permet à l'apprenant d'aller à son rythme, jusqu'au bout de sa réflexion, de rassembler ses idées et celles des autres pour les argumenter, et de les accorder dans un ensemble logique et cohérent ; ces processus sont plus exigeants et formatifs qu'une contribution orale improvisée dans un cadre présentiel où le délai de réflexion et le temps de parole sont très limités.

\section{- Le message écrit privilégie le contenu et équilibre les relations entre les participants :}

L'attention des participants, lors de l'intervention en face à face, est d'emblée accaparée par les caractéristiques physiques (taille, voix, physionomie, esthétique...) et les indices sociaux (sexe, accent, timidité...) de l'intervenant : sa physionomie peut donner une impression d'assurance ou d'hésitation, l'intonation de la voix peut souligner certains points de son exposé ou en masquer les faiblesses. De plus, en présence des pairs et de l'enseignant, l'apprenant, peu assuré (par manque de formation antérieure ou par timidité), hésite à s'exprimer. En revanche, le même apprenant, collaborant en ligne, 
n'hésite pas à s'exprimer en mode asynchrone qui lui permet de réfléchir, de lui fournir des informations complémentaires. De même, il peut demander d'aide ou d'avis.

\section{- Convivialité dans l'apprentissage collaboratif :}

Une convivialité doit être suscitée dans l'apprentissage collaboratif en ligne : au début de formation, le formateur affiche dans l'espace virtuel réservé à son groupe la liste de ses membres de participants, sa propre auto-présentation et un message pour inviter chaque participant à y afficher la sienne. Chacun y signale sa formation, son expérience, ses environnements géographique, professionnel, social et culturel, ainsi que les motivations pour cette formation.

\section{- Effet d'émulation, d'entraînement et d'entraide :}

La spontanéité des interactions dans l'apprentissage collaboratif en ligne au sein de petits groupes d'apprenants ( 8 participants au maximum) dans les activités communes produit un sentiment de solidarité, un esprit d'entraide et d'encouragement mutuel entre les apprenants. Une familiarité se crée ainsi entre apprenants en ligne, malgré les différences d'âge, de culture, de formation initiale et de profession, malgré la distance ou grâce à celle-ci et aux différences entre apprenants. Cet état rend les travaux des groupes d'apprenants à distance très animés et très productifs. De plus, la contribution des pairs suscite l'intérêt de chacun, une émulation et des effets d'entraînement efficaces.

- La permanence des contributions stimule la production et permet la « mutualisation » et l'évaluation :

Dans l'apprentissage collaboratif présentiel, les plus confiants participent à parler, mais la majorité préfère s'abstenir. Dans l'apprentissage collaboratif en ligne, la permanence des contributions des participants en voyant les contributions de ses pairs s'accumuler de jour en jour d'abord par émulation, ensuite pour l'évaluation (par les pairs et par le formateur) stimule la production de tous les participants. 


\section{- Capacité de supervision des tuteurs décuplée :}

Les formateurs ou tuteurs d'apprentissage collaboratif ont la possibilité de superviser en ligne un plus grand nombre de petits groupes qu'ils ne peuvent pas le faire en présentiel. Grâce aux moyens de communication synchrone ou asynchrone, la collaboration entre les formateurs est plus facile en ligne qu'en présentiel. L'économie de temps et de déplacements résultant de la flexibilité de l'apprentissage collaboratif en ligne permet aux formateurs de quintupler la capacité d'animation et de supervision des apprentissages en ligne par rapport aux mêmes fonctions en présentiel.

Ainsi, les apprenants, dans le travail collaboratif en ligne, se trouvent dans un climat pédagogique favorable à l'apprentissage car ils déterminent par eux-mêmes les horaires du travail, la manière d'exécuter les activités. De même, ils trouvent des ressources pédagogiques nombreuses et variées, des outils et des applications innovateurs... . Ce type d'apprentissage rend l'apprenant plus ouvert et plus tolérant envers les différences car il présente de l'aide à ses pairs et accepte de recevoir de l'aide de la part d'eux.

Pour suivre la méthode de l'apprentissage collaboratif en ligne, les apprenants et le tuteur doivent suivre trois phases essentielles : former les équipes, exécuter les tâches, évaluer l'apprentissage. Bennett (2003) détermine ces trois phases ainsi :

- Former des équipes et sélectionner un projet : Les étudiants peuvent choisir parmi un certain nombre de projets identifiés par l'instructeur avant la session ou déterminés par eux-mêmes. La condition est que chaque projet doit impliquer un problème d'éducation ou de formation à résoudre. Les étudiants commencent alors le processus d'analyse et d'exploration des problèmes de leur projet. Ce processus a deux aspects: rechercher leur projet à travers les discussions, tout en négociant la répartition du travail et des responsabilités entre les membres de l'équipe et analyser les activités de discussion qui encouragent les apprenants à relier leur compréhension des cas à leurs propres expériences, aux concepts qu'ils ont 
rencontrés dans la littérature et à leurs idées initiales concernant leurs propres projets.

- Commencer à se concentrer sur les tâches collaboratives en préparant un énoncé de conception qui détaille la solution proposée tout en s'engageant dans des activités authentiques telles que les activités dans lesquelles les apprenants font face à des défis cognitifs.

- É valuer et réfléchir aux résultats du projet et aux expériences. La tâche de réflexion comporte deux volets : une réponse individuelle à une série de questions focales et la préparation d'un cas collaboratif qui «raconte l'histoire» du projet pour vérifier la compréhension individuelle et collective. Les résultats des évaluations sont utilisés pour affiner la version finale du matériel et des activités du sujet.

Ces phases prédéterminées représentent les étapes essentielles de l'apprentissage collaboratif en ligne. Chaque équipe de travail peut élaborer sa propre stratégie d'enseignement collaboratif en ligne. Toutefois, il y a des facteurs de réussite pour cette méthode d'apprentissage. Ces facteurs ont un impact significatif sur la propension à collaborer dans l'apprentissage en ligne Jeunesse et Dumont (2004) en proposent quatre :

\section{- Le comportement du tuteur :}

Le tuteur n'a pas seulement des fonctions organisationnelles, mais il doit favoriser le sentiment d'appartenance à un groupe, encourager la persévérance et le soutien motivationnel. Le tuteur doit, également, susciter l'émergence de « la confiance en soi » auprès les apprenants et leur reconnaissance au sein du groupe.

\section{- L'intégration et l'articulation des outils de communication et des modalités de collaboration dans la scénarisation pédagogique :}

Le scénario pédagogique doit déterminer quel outil doit être utilisé pour quelle activité. Le scénario doit, également, expliciter les modalités de production, de co-négociation, de co-réalisation etc.). 


\section{- L'importance de la constitution des équipes :}

Le travail collaboratif prend la forme horizontale du travail ; au sein de la construction collective d'une œuvre commune, une personne régule l'action de l'autre et les rôles peuvent s'inverser au cours de l'interaction. Cette construction nécessite une confiance mutuelle, un relatif équilibre de compétences et un engagement mutuel des participants, des valeurs et des stratégies communes.

\section{- Réfléchir l'évaluation en tant que processus de formation :}

L'évaluation, dans l'apprentissage collaboratif en ligne, doit être organisée et planifiée d'une manière claire et précise. Le tuteur doit bien réfléchir le processus d'évaluation en planifiant le cursus de formation en ligne. De même, il est nécessaire de prendre garde aux tentatives de tromperies et de tricheries de certains apprenants. L'évaluateur doit garantir une certaine transparence dans la performance de chaque apprenant et intervenir efficacement dans les feed-back aux apprenants.

Ainsi, la méthode de l'apprentissage collaboratif en ligne présente des avantages très nombreux, à condition qu'elle revête un certain nombre de mesures qui sont les suivantes :

- Choisir les outils adéquats pour atteindre les objectifs d'apprentissage ;

- Contribuer à augmenter la confiance en soi des apprenants et le sentiment de sécurité entre les participants ;

- Répartir les groupes de travail selon leurs intérêts et leurs besoins ;

- Augmenter le sentiment d'appartenance auprès les apprenants envers le groupe de travail et toute la classe ;

- Préparer un plan de travail et un scénario d'apprentissage collaboratif ;

- Présenter de l'aide aux apprenants à l'égard des outils et des applications de l'apprentissage collaboratif en ligne en cas de besoin ; 
- Encourager les apprenants à participer dans les activités de travail collaboratif ;

- Adopter l'évaluation continue (évaluation diagnostique, évaluation formative, évaluation sommative d'étape et évaluation sommative finale).

En profitant de la flexibilité temporelle et spatiale, des ressources disponibles, des activités de travail collaboratif en ligne et du tutorat $\mathrm{du}$ formateur, les apprenants envisagent une méthode d'apprentissage prometteuse, ils deviennent plus actifs dans l'apprentissage, plus capables de présenter de l'aide à leurs collègues, plus tolérants envers leurs compétences puisqu'ils acceptent d'être aidés par les pairs ; ils peuvent, ainsi, atteindre les objectifs du travail visés

\section{Méthodologie :}

\section{Elaborer le pré/post-test :}

La recherche présente vise à utiliser un dispositif d'apprentissage collaboratif en ligne pour développer la compréhension et la production des expressions idiomatiques. Pour atteindre cet objectif, le chercheur a élaboré un dispositif basé sur les outils collaboratifs de Google. Pour mesurer l'efficacité de ce dispositif, le chercheur a élaboré un pré/post-test.

\section{Source du test}

Pour élaborer le pré/post-test des expressions idiomatiques, le chercheur a eu recours aux sites internet suivants :

https://www.bonjourdefrance.com/exercices/expressions-imagees/

https://www.francaisfacile.com/exercices/exercice-francais-

2/exercice-francais-3924.php

http://peinturefle.free.fr/activite/exression.htm

https://ticsenfle.blogspot.com/2015/06/expressions-idiomatiquesjeux-et.html 


\section{Description du test :}

Le pré/post-test se compose de 50 items de questions à choix multiples. Chaque item contient 4 choix dont l'un est seulement correct. Ce test se compose de deux parties : la première partie porte sur la compréhension des expressions idiomatiques (25 items) et la deuxième partie porte sur la production des expressions idiomatiques (25 items).

\section{Validité et fidélité du test :}

Pour mesurer la validité et la fidélité du test, le chercheur a fait passer le test auprès 20 étudiants. Le chercheur a vérifié la validité du test par l'utilisation du coefficient alpha de Kuder Richardson-20. Le résultat obtenu (0.82) a indiqué que le test est valide

Pour calculer la fidélité du test, nous avons administré le test deux fois, le premier mars 2020 puis deux semaines après. Nous avons calculé le coefficient de corrélation entre les deux passations en appliquant la formule statistique suivante :

$$
\square=\frac{\mathrm{n} \sum \mathrm{xy}-\left(\sum \mathrm{x}\right)\left(\sum \mathrm{y}\right)}{\sqrt{\left(\mathrm{n} \sum \mathrm{x} 2-\left(\sum \mathrm{x}\right) 2-\left(\mathrm{n} \sum \mathrm{y} 2-\left(\sum \mathrm{y}\right) 2\right.\right.}}
$$

$\mathrm{R}=$ coefficient de corrélation

$\mathrm{N}=$ nombre des étudiants

$\mathrm{T}=$ total des notes des étudiants

$\mathrm{X}=$ notes de la $1^{\text {ère }}$ passation

$\mathrm{Y}=$ notes de la $2^{\text {ème }}$ passation

Le résultat de cette équation a montré que le coefficient de corrélation entre les deux passations du test est 0.79 . Ce résultat affirme que notre test est fidèle.

\section{Durée du test :}

Le chercheur a calculé la durée du test par la formule suivante :

Durée $=$ Temps total mis par chaque étudiant/Nombre des étudiants. Durée $=900 / 20=45$ minutes

Donc, la durée du test est 45 minutes 


\section{Elaboration du dispositif :}

Pour élaborer le dispositif d'apprentissage en ligne, le chercheur a suivi les étapes suivantes :

\section{Déterminer les objectifs du dispositif :}

Objectifs généraux du dispositif :

Le dispositif d'apprentissage proposé vise essentiellement à :

- Développer la compréhension des expressions idiomatiques en français langue étrangère ;

- Développer la production des expressions idiomatiques en français langue étrangère ;

\section{Objectifs spécifiques du dispositif :}

A la fin du dispositif proposé, les participants doivent être capables de :

- Comprendre la signification des expressions idiomatiques les plus utilisées en français ;

- Utiliser les expressions idiomatiques les plus fréquentes en français.

- Donner des exemples sur les expressions idiomatiques les plus utilisées en français ;

- Raconter les origines des expressions idiomatiques les plus utilisées en français ;

- Donner les équivalences arabes des expressions idiomatiques les plus utilisées en français.

\section{Déterminer le contenu du dispositif :}

Le chercheur a déterminé les expressions idiomatiques les plus utilisées en français en s'appuyant sur la littérature, les études antérieures dans ce domaine et les sites internet les plus fiables. Le dispositif proposé contient 10 unités.

Unité 0 : Briser la glace

Cette unité vise à familiariser les participants à l'utilisation des outils et des applications de l'apprentissage collaboratif en ligne. De même, elle vise à présenter les objectifs et les procédures à suivre dans le dispositif.

5 unités sur les expressions idiomatiques concernant le mot science, les couleurs, les fruits et légumes, les mots de la nature et les parties du corps.

4 unités sur des expressions idiomatiques variées. 


\section{Types d'activités dans le dispositif :}

\section{Activité 1. Lecture collaborative}

Objectifs de l'activité :

- Déterminer les expressions idiomatiques dans le texte ;

- Définir les significations des expressions idiomatiques citées dans le texte ;

- Donner des exemples pour l'utilisation des expressions idiomatiques.

Outil de l'activité : Google Meet, Blog

Durée de l'activité : 60 minutes

Déroulement de l'activité :

- Un des participants lit le texte ;

- Un autre participant détermine les expressions idiomatiques ;

- Le leader demande aux participants la signification de chaque expression ;

- Si les participants ne connaissent pas la signification de ces expressions, le leader demande à un des participants de rechercher la signification des expressions sur l'internet ;

- Le leader demande, ensuite, aux participants de donner des exemples pour l'utilisation de ces expressions.

\section{Activité 2.}

Chaque groupe recherche les expressions idiomatiques en lien avec un sujet donné, leurs significations et leurs origines si c'est possible.

\section{Activité 3.}

Chaque groupe fait une conversation sur le résultat de la recherche exécuté pendant l'acticité 2. Les participants doivent utiliser l'application Google Meet et le tuteur participe avec chaque groupe dans leur conversation. 


\section{Activité 4. Ecriture collaborative :}

- Chaque groupe se collabore pour rédiger un article, un rapport sur les expressions idiomatiques retrouvées dans l'activité 2. Chaque membre de groupe peut ajouter des passages et des phrases ou commenter ce que ses collègues ont rédigé.

\section{Déterminer les outils du web utilisés dans le dispositif :}

Les outils du web qu'on peut utiliser dans l'apprentissage collaboratif sont très nombreux et variés. Le chercheur a choisi les outils et les applications de Google car ils sont gratuits et disponibles pour tous les participants. Les outils et les applications choisis sont les suivants :

\section{Blog :}

Pour présenter les textes et les documents sur les expressions idiomatiques, le chercheur a créé un blog en utilisant le site blooger.com. Dans ce blog, les participants peuvent lire des informations sur les expressions idiomatiques, leurs utilisations et parfois leurs origines. De même, les participants peuvent ajouter des commentaires sur les textes présentés. Le lien du blog est https://mohamedgomaar.blogspot.com/

\section{Google Classroom :}

Le chercheur a créé une classe virtuelle sur Google Classroom (https://classroom.google.com/c/MTUxOTA4NDA2ODQ4), il a invité les apprenants à rejoindre cette classe à travers les e-mails (Gmail). Avant de commencer de travailler dans la classe virtuelle, le chercheur a demandé aux participants de se présenter comme une étape de biser la glace.

\section{Google docs}

Cet outil est utilisé dans les activités de l'écriture collaborative. C'est une application qui permet d'éditer des textes en ligne en les sauvegardant automatiquement, de les stocker, de les partager. Cet outil permet de collaborer dans la création des textes, d'accéder aux documents des collègues et y rédiger des commentaires ou modifier quelques passages. 


\section{Google forms}

C'est une application qui permet d'élaborer des tests et des questions automatiquement corrigés. De même, elle peut créer des statistiques et des figures pour les réponses aux questions.

\section{Calendrier Google}

Le calendrier de Google permet d'enregistrer les horaires et les rendez-vous des activités d'apprentissage synchrones ou asynchrones. Il peut être connecté aux comptes Gmail pour informer les participants sur les activités et leurs horaires.

\section{Rédiger les scénarios du dispositif :}

Le chercheur a rédigé le scénario selon lequel vont dérouler les séances d'apprentissage collaboratif: objectifs des séances, les activités d'apprentissage collaboratifs, les activités d'enseignement/apprentissage synchrones (visioconférence ou clavardage (chat)) et celles asynchrones (écriture collaborative sur Google docs). Le scénario détermine les détails du dispositif, les outils à utiliser, les différentes activités, les questions à poser et un modèle de leurs réponses.

\section{Évaluer le dispositif :}

Pour évaluer le dispositif et déterminer les points forts et les points à améliorer, le chercheur a appliqué une partie du dispositif sur un petit nombre (10 participants). Quelques modifications ont été faites à la lumière de l'application préliminaire du dispositif.

\section{Expérimenter le dispositif :}

L'expérimentation du dispositif proposé a eu lieu pendant le premier semestre de l'année universitaire 2020/2021 pendant les deux mois de novembre et décembre. Cette expérimentation est passée par plusieurs étapes:

- Déterminer le design expérimental et sélectionner les participants parmi les étudiants de la 4ème année de la faculté de pédagogie de Minia ; 
- Faire passer le pré-test des expressions idiomatiques auprès du groupe expérimental et du groupe témoin ;

- Expérimenter les activités de l'apprentissage collaboratif en ligne sur le groupe expérimental ;

- Faire passer le post-test des expressions idiomatiques auprès du groupe expérimental et du groupe témoin ;

- Analyser les résultats ;

\section{Déroulement des activités d'apprentissage collaboratif en ligne :}

Dans notre dispositif, les activités sont subdivisées en différentes périodes :

\section{Classement des groupes :}

Le tuteur a demandé aux participants de se diviser en des groupes de cinq. Chaque groupe détermine sa manière de travail et ses horaires des activités synchrones et des activités asynchrones selon leur temps et leur besoin. De même, il y a des rencontres pour tous les participants pour présenter ce que chaque groupe a réalisé dans les activités demandées.

\section{Lecture collaborative :}

Cette stratégie est très proche de la stratégie de l'enseignement réciproque, elle vise à collaborer dans la lecture et la compréhension d'un texte contenant des expressions idiomatiques. Les participants se divisent en groupes de 5: le leader, le lecteur, le scripteur, le chercheur et l'interprète. Le leadeur demande au lecteur de lire à haute voix le premier paragraphe et demande au scripteur d'écrire les expressions idiomatiques contenues dans le paragraphe. Une discussion autour de la signification de ces expressions est faite entre les membres du groupe. Si tous les membres ne connaissent pas la signification des expressions idiomatiques présentées, le leadeur demande au chercheur de chercher la signification sur l'internet et demande à l'interprète de donner l'équivalence en arabe et des exemples sur les expressions. Dans cette activité, les participants utilisent deux outils : Google Meet pour faire la réunion sur l'internet et le blog créé par le chercheur pour lire les textes visés. 


\section{Conversation :}

Les participants utilisent l'application Google Meet pour faire des conversations sur les expressions idiomatiques de leur sujet. Chaque groupe fait une conversation sur le résultat de la recherche exécutée pendant l'activité de la recherche sur la signification des expressions idiomatiques. Le tuteur participe avec chaque groupe dans leur conversation.

\section{Production collaborative :}

Dans cette phase, les participants produisent un document collectif de synthèse. Chaque groupe se collabore pour rédiger un article, un rapport sur les expressions idiomatiques retrouvées. Les participants utilisent Google Docs pour se collaborer dans la rédaction de l'article ou du rapport. Chaque membre de groupe peut ajouter des passages ou commenter ce que ses collègues ont rédigé.

\section{L'évaluation dans le dispositif :}

L'évaluation dans le dispositif suit deux types d'évaluation: l'évaluation formative à travers les questions posées pendant les séances et l'évaluation sommative à travers le pré/post-test.

\section{Résultats de la recherche :}

Après avoir fini l'expérimentation du dispositif proposé qui a eu lieu de 24 octobre à 3 décembre 2020 et administré le post-test des expressions idiomatiques sur les groupes de la recherche, le chercheur fait l'analyse statistique en utilisant le programme statistique SPSS (version 23) et il utilise " $\mathrm{T}$ " test pour calculer la moyenne arithmétique, l'écart type et la valeur de "t".

\section{Vérification de l'égalité des deux groupes avant l'expérimentation :}

Pour vérifier l'égalité des deux groupes expérimental et témoin avant l'expérimentation, le chercheur a calculé la moyenne arithmétique, l'écart type et la valeur de T. Les deux tableaux suivants résument les résultats obtenus : 
Tableau 1 :

Les résultats du pré-test des deux groupes (expérimental et témoin)

Compréhension des expressions idiomatiques

\begin{tabular}{cccccc}
\hline Groupe & $\mathrm{N}$ & MA & ET & T & S. ou N.S. \\
Expérimental & $3 \cdot$ & 7.6 & 2.6 & & \\
Témoin & 27 & 7.18 & 1.5 & .709 & Non significative \\
\hline
\end{tabular}

$\mathrm{N}=$ nombre des étudiants $\mathrm{MA}=$ moyenne arithmétique $\mathrm{ET}=$ écart type $\quad \mathrm{T}=$ valeur de " $\mathrm{t}$ " test $\mathrm{S}=$ significative $\mathrm{NS}=$ non significative

Tableau 2:

Les résultats du pré-test des deux groupes (expérimental et témoin)

Production des expressions idiomatiques

\begin{tabular}{cccccc}
\hline Groupe & $\mathrm{N}$ & $\mathrm{MA}$ & $\mathrm{ET}$ & $\mathrm{T}$ & S. ou N.S. \\
Expérimental & 30 & 5.8 & 2.26 & & \\
Témoin & 27 & 5.4 & 1.08 & .820 & Non significative \\
\hline
\end{tabular}

Les résultats obtenus montent qu'il n'y a pas de différences significatives entre les moyennes des notes du groupe expérimental et celles du groupe témoin au pré-test. Ce qui affirme l'égalité des deux groupes expérimental et témoin avant l'expérimentation.

\section{Vérification de la première hypothèse}

« Il y a une différence statistiquement significative entre la moyenne des notes des étudiants du groupe expérimental au pré-post test concernant la compréhension des expressions idiomatiques en français langue étrangère en faveur du post-test. »

Pour vérifier cette hypothèse, le chercheur a calculé la moyenne arithmétique, l'écart type et la valeur de T. Le tableau suivant résume les résultats obtenus : 


\section{Tableau 3}

: La différence entre le pré-test et le post-test du groupe expérimental (Compréhension des expressions idiomatiques)

\begin{tabular}{ccccccc}
\hline Test & N & MA & ET & T & S. ou N.S. & VI \\
Pré-test & 30 & 7.6 & 2.66 & 41.7 & $\begin{array}{c}\text { Significative au } \\
\text { degré } 0.01 \text { en faveur } \\
\text { de la post-application }\end{array}$ & 0.98 \\
Post-test & & 20.86 & 1.85 & & re
\end{tabular}

Les résultats dans le tableau montrent qu'il y a une différence statistiquement significative entre la moyenne des notes du groupe expérimental au pré-test et celle au post-test (Compréhension des expressions idiomatiques) en faveur du post-test. La valeur de $\mathrm{T}$ calculé est plus grande que celle du tableau au niveau de $p>0,01$. La valeur de l'influence est 0.98 ; c'est un taux très élevé. Ce qui vérifie la vérité de la première hypothèse de la recherche et affirme l'efficacité du dispositif proposé pour développer la compréhension des expressions idiomatiques en français langue étrangère auprès les étudiants de la $4^{\text {ème }}$ année de la faculté de pédagogie de Minia.

\section{Vérification de la deuxième hypothèse}

« Il y a une différence statistiquement significative entre la moyenne des notes des étudiants du groupe expérimental au pré-test et celle au post-test concernant la production des expressions idiomatiques en français langue étrangère en faveur du post-test. »

Pour vérifier cette hypothèse, le chercheur a calculé la moyenne arithmétique, l'écart type et la valeur de T. Le tableau suivant résume les résultats obtenus :

\section{Tableau 4 :}

La différence entre le pré-test et le post-test du groupe expérimental (Production des expressions idiomatiques)

\begin{tabular}{ccccccc}
\hline Test & N & MA & ET & T & S. ou N.S. & VI \\
Pré-test & 30 & 5.8 & 2.26 & 33.4 & $\begin{array}{c}\text { Significative au degré } \\
0.01 \text { en faveur de la } \\
\text { post-application }\end{array}$ & 0.97 \\
Post-test & & 19.8 & 1.3 & & con & \\
\hline
\end{tabular}


Les résultats obtenus montrent qu'il y a une différence statistiquement significative entre la moyenne des notes du groupe expérimental au pré-test et celle au post-test (Production des expressions idiomatiques) en faveur du post-test. La valeur de $\mathrm{T}$ calculé est plus grande que celle du tableau au niveau de $p>0,01$. La valeur de l'influence est 0.97 ; c'est un taux très élevé. Ce qui vérifie la vérité de la deuxième hypothèse de la recherche et affirme l'efficacité du dispositif proposé pour développer la production des expressions idiomatiques en français langue étrangère auprès les étudiants de la $4^{\text {ème }}$ année de la faculté de pédagogie de Minia.

\section{Différences entrele pré-test et le post-test du groupe expérimental}

25

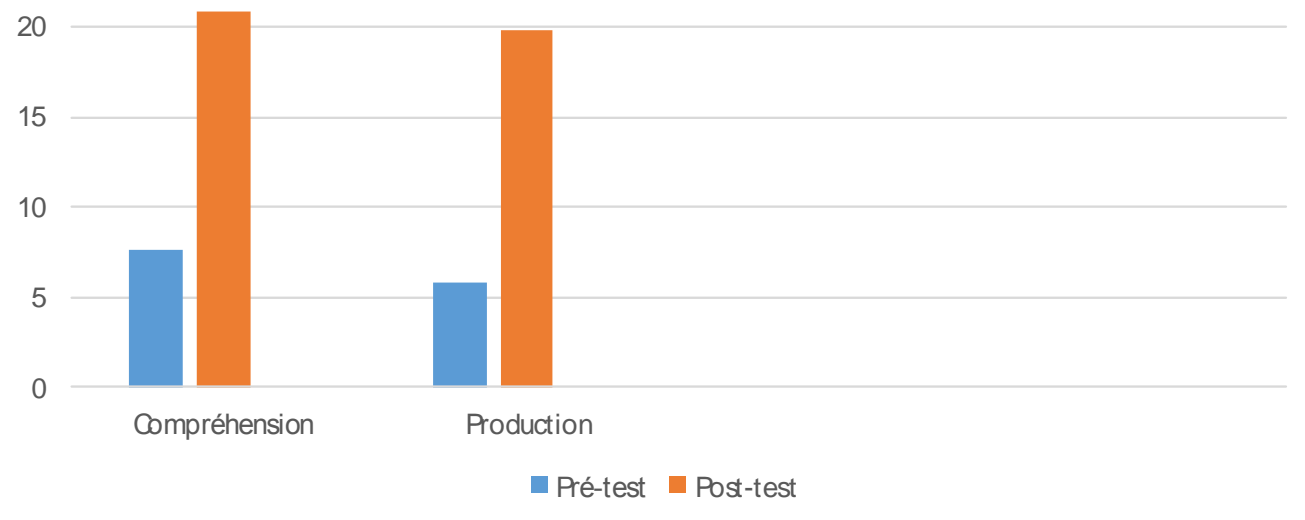

\section{Vérification de la troisième hypothèse}

«Il y a une différence statistiquement significative entre la moyenne des notes des étudiants du groupe expérimental et celle du groupe témoin au post-test concernant la compréhension des expressions idiomatiques en français langue étrangère en faveur du groupe expérimental. »

Pour vérifier cette hypothèse, le chercheur a calculé la moyenne arithmétique, l'écart type et la valeur de T. Le tableau suivant résume les résultats obtenus : 


\section{Tableau 5 :}

Les résultats du post-test des deux groupes expérimental et témoin (Compréhension des expressions idiomatiques)

\begin{tabular}{|c|c|c|c|c|c|c|}
\hline Groupe & $\mathrm{N}$ & MA & ET & $\mathrm{T}$ & S. ou N.S. & VI \\
\hline Expérimental & 3 . & 20.8 & 1.85 & 18.68 & $\begin{array}{c}\text { Significative au } \\
\text { degré } 0.01 \text { en }\end{array}$ & 0.92 \\
\hline Témoin & 27 & 9.18 & 2.82 & & $\begin{array}{c}\text { faveur de la post- } \\
\text { application }\end{array}$ & \\
\hline
\end{tabular}

Les résultats obtenus indiquent qu'il y a une différence statistiquement significative entre la moyenne des notes du groupe expérimental et celle du groupe témoin au post-test. La valeur de $\mathrm{T}$ calculé est plus grande que celle du tableau au niveau de $p>0,01$. Ce qui vérifie la vérité de la troisième hypothèse de la recherche et affirme l'efficacité du dispositif proposé pour développer la compréhension des expressions idiomatiques en français langue étrangère auprès les étudiants de la $4^{\text {ème }}$ année de la faculté de pédagogie de Minia.

\section{Vérification de la quatrième hypothèse}

«Il y a une différence statistiquement significative entre la moyenne des notes des étudiants du groupe expérimental et celle du groupe témoin au post-test concernant la production des expressions idiomatiques en français langue étrangère en faveur du groupe expérimental. »

Pour vérifier cette hypothèse, le chercheur a calculé la moyenne arithmétique, l'écart type et la valeur de T. Le tableau suivant résume les résultats obtenus :

\section{Tableau 6 :}

Les résultats du post-test des deux groupes expérimental et témoin (Production des expressions idiomatiques)

\begin{tabular}{|c|c|c|c|c|c|c|}
\hline Groupe & $\mathrm{N}$ & MA & ET & $\mathrm{T}$ & S. ou N.S. & VI \\
\hline Expérimental & 3 . & 19.8 & 1.3 & 28.7 & $\begin{array}{l}\text { Significative au } \\
\text { degré } 0.01 \text { en }\end{array}$ & 0.96 \\
\hline Témoin & 27 & 7.1 & 2.0 & & $\begin{array}{l}\text { faveur de la post- } \\
\text { application }\end{array}$ & \\
\hline
\end{tabular}

\section{9}


Les résultats obtenus dans le tableau précédent montrent qu'il y a une différence statistiquement significative entre la moyenne des notes du groupe expérimental et celle du groupe témoin au post-test. La valeur de $T$ calculé est plus grande que celle du tableau au niveau de $p>0,01$. Ce qui vérifie la vérité de la quatrième hypothèse de la recherche et affirme l'efficacité du dispositif proposé pour développer la production des expressions idiomatiques en français langue étrangère auprès les étudiants de la $4^{\text {ème }}$ année de la faculté de pédagogie de Minia.

\section{Différences entre le groupe expérimental et le groupe témoin dansle post-test}

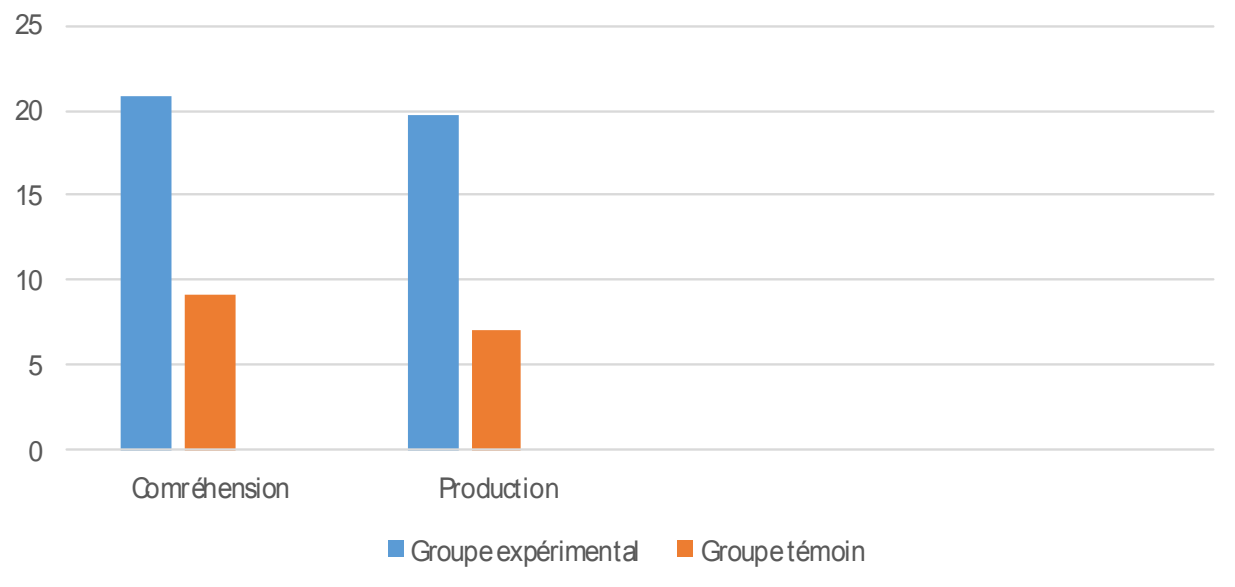

\section{Discussion des résultats :}

En se basant sur les résultats présentés dans les pages précédentes, on remarque que le dispositif proposé basé sur la collaboration en ligne est efficace dans le développement de la compréhension et la production des expressions idiomatiques en français langue étrangère auprès les étudiants de la $4{ }^{\text {ème }}$ année de la faculté de pédagogie de Minia. Ces résultats peuvent être attribuables à la richesse des activités qui rendent les apprenants plus actifs à participer, à accéder à des ressources inépuisables et à se collaborer pour générer les connaissances. De même, les apprenants, dans ce dispositif proposé, se sentent responsables de leur apprentissage et de 
l'apprentissage de leurs pairs. Les participants ont admiré beaucoup les applications et les outils utilisés dans ce dispositif proposé qui rendent les processus d'enseignement/apprentissage plus flexibles et plus créatifs; les participants peuvent organiser les activités selon leur temps et leur besoin. Les apprenants, dans les activités d'apprentissage collaboratif en ligne, se trouvent dans un climat favorable à l'apprentissage grâce aux groupes de travail collaboratif ; ils deviennent, alors, plus motivés à déployer plus d'efforts à acquérir les expressions idiomatiques.

\section{Recommandations :}

A la lueur des résultats de la recherche, le chercheur présente les recommandations suivantes :

- Il est nécessaire d'utiliser les outils et les applications de l'apprentissage collaboratif en ligne pour développer la compréhension des expressions idiomatiques ;

- Il est nécessaire d'utiliser les outils et les applications de l'apprentissage collaboratif en ligne pour développer la production des expressions idiomatiques ;

- Il est nécessaire d'utiliser les outils et les applications de l'apprentissage collaboratif en ligne dans l'apprentissage à distance surtout les outils de Google ;

- Il est nécessaire de faire des stages pour les étudiants de la faculté de pédagogie pour utiliser les outils et les applications de l'apprentissage collaboratif en ligne dans les processus d'enseignement/apprentissage.

\section{Suggestions :}

A la lueur des résultats de cette recherche, le chercheur présente les suggestions suivantes :

- Utiliser l'apprentissage collaboratif en ligne pour développer les compétences de la compréhension écrite ;

- Utiliser 1'apprentissage collaboratif en ligne pour développer les compétences de la production écrite ; 
- Utiliser l'apprentissage collaboratif en ligne pour développer les compétences de la compréhension orale ;

- Utiliser 1'apprentissage collaboratif en ligne pour développer les compétences de la production orale.

\section{Conclusion :}

Les expressions idiomatiques sont très importantes dans la communication avec les locuteurs natifs parce qu'elles représentent un aspect essentiel de la culture d'une nation. Toutefois, les expressions idiomatiques sont restées pendant longtemps presque absentes dans les programmes de formation et dans les méthodes d'apprentissage du FLE. Dans la recherche présente, le chercheur a élaboré un dispositif d'apprentissage en ligne pour développer la compréhension et la production des expressions idiomatiques. Ce dispositif est basé sur la collaboration en ligne.

Le chercheur a choisi le contenu du dispositif parmi les expressions idiomatiques les plus utilisées en français. Les résultats ont montré l'efficacité du dispositif proposé d'apprentissage collaboratif en ligne dans le développement de la compréhension et la production des expressions idiomatiques en français langue étrangère auprès les étudiants de la $4^{\text {ème }}$ année de la faculté de pédagogie de Minia.

Le dispositif proposé contient des activités synchrones et asynchrones ; ces activités passent par certaines étapes et procédures : former les groupes, lire d'une manière collaborative, faire de recherches en ligne, faire des conversations, écrire d'une manière collaborative. Au début de l'expérimentation, les participants dans ce dispositif étaient moins actifs à participer dans les activités et moins confiants. Dans les séances ultérieures, les participants deviennent très actifs à participer dans les différentes activités. Ils ont collaboré d'une bonne manière dans l'exécution des activités d'apprentissage. 


\section{Références}

Alwadi, A. et Alhathal, B. (2013). Les expressions idiomatiques en classe de FLE, Analyses et Propositions. J. King Saud Univ., Vol. 25, Lang. \& Tran. (Special Issue), pp. 1523, Riyadh (2013/1434H.)

BenabdAllah, M. ; Derraz, M. et Legssyer, M. (2013).Travaux collaboratifs de recherche doctorale basés sur l'eLearning. Dossiers de Recherches en Economie et

Gestion, numéro 2 : Juin 2013, p. 137-148.

Bennett, S. (2003). Supporting Collaborative Project Teams Using Computer-Based Technologies. In Online collaborative learning: theory and practice / Tim S. Roberts, Editor. Information Science Publishing, London and Information Science Publishing, Hershey.

Cáceres-Guerrero, L. D. (2017). "Transfert dans l'acquisition des expressions idiomatiques en français langue étrangère". Rastros Rostros 19.35, 1-15. Web. doi: https://doi. org/10.16925/2382-4921.2017.35.06.

Cavalla, C., \& Labre, V. (2009). L'enseignement en FLE de la phraséologie du lexique des affects. In Tutin A. \& Novakova I. (Eds.), Le lexique des émotions et sa combinatoire lexicale et syntaxique (pp. 297-316). Grenoble : Ellug.

CECRL (Cadre Européen Commun de Référence pour les Langues). (2001). apprendre, enseigner, évaluer. Conseil de L'Europe.

Chiong, R. and Jovanovic, J. (2012). Collaborative Learning in Online Study Groups: An Evolutionary Game Theory Perspective. Journal of Information Technology Education: Research Volume 11, 2012, p. 81-101.

Da Silva, G. et Ponge, R. (2012). Les expressions idiomatiques et les difficultés de compréhension et de traduction du FLE. Synergies Brésil n 10 - 2012 pp. 113-122.

De Serres, L. (2011). Tendances en enseignement des expressions idiomatiques en langue seconde : de la théorie à la 
pédagogie Revue canadienne de linguistique appliquée, numéro hors-série : 14,2 (2011) : 129-155.

Denis, C. (2005). Travail en équipe et apprentissage collaboratif dans un climat de grande compétitivité. Collège de Sherbrooke, Québec, Canada ISBN 2-920-916-56-4.

Dewaele, J.-M. et Regan, V. (2002). Maîtriser la norme sociolinguistique en interlangue française : le cas de l'omission variable de "ne" Journal of French Language Studies, 12, 2, 123-148.

Dictionnaire Larousse (version électronique). https://www.larousse.fr/dictionnaires/francais/phras\%C 3\%A9ologie/60535.

Dictionnaire le Petit robert (1991).Le français langue seconde, origines d'une notion et implications didactiques. Paris, Hachette.

Dubois, J. (1993).Dictionnaire de linguistique Larousse. Paris. Ehegôtz E., (1990). Versuch einer Typologie von Entsprechungen im zweisprachigen phraseologischen Wôrterbuch. Zeitschrift für Slawistik, 35/4.

Frémont, L. (2011). Quelle stratégie pour la mise en place d'une plateforme collaborative de partage de l'information au sein d'une organisation? Le cas d'un site SharePoint pour la Division santé d'Orange-Orange Healthcare. domain_shs.info.docu. 2011. mem_00679465.

Gonzalez-Rey, I. (2016). Une approche analogique à la compétence phraséologique: une double compétence, intégrale et intégrée. Language Design, Issue Spécial (2016: 165188)

Hallsteinsdóttir, E. (In print). Phraseology and foreign language learning. In: Szerszunowicz, Joanna (eds.): Linguoculturalresearch on phraseology: Intercontinental Dialogue on Phraseology 3. Bialystok: Wydawnictwo Uniwersytetu w Białymstoku, reçu de http://hallsteinsdottir.org/wpcontent/uploads/2016/11/Hallsteinsdottir-2016Phraseologie-and-foreign-language-learning.pdf 
Hattouti, J. et Gil, S. (2016). Le développement de la compréhension des expressions idiomatiques : une revue de littérature. L'année psychologique, 2016/1 (Vol. 116), pages 105 à 136.

Holtzer, G., 2002. « S'approprier une langue étrangère dans des situations et des dispositifs pluriels ». In Appropriation des langues au Centre de la Recherche. Frankfurt am Main : Peter Lang, p. 14-17.

Khoshhal, Y., \& Hassasskhah (2017).The Effect of Explicit Teaching of Idioms on Strategy Choice for EFL Learners in a Reading Comprehension Test. ReiDoCrea, 6, 8494.http://hdl.handle.net/10481/45028

Ilie, O.-A. (2019). The intercultural competence. Developing effective intercultural communication skills. International Conference KNOWLEDGE-BASED ORGANIZATION Vol. XXV No 2019.

Jeunesse, C. et Dumont, C. (2004). Une pédagogie pour susciter l'apprentissage collaboratif en ligne. Information Sciences for Decision Making, $\mathrm{N}^{\circ} 18$, reçu de http://isdm.univ-tln.fr/PDF/isdm18/38-jeunessedumont-arnaud.pdf

Johnson, D. W. (1994). The New Circles of Learning: Cooperation in the Classroom and School. Alexandria: Association for Supervision and Curriculum development.

Juan, S. et Zhihong, P. (2018). Pour une approche sociolinguistique en didactique du français langue étrangère - l'argot français contemporain en classe. Synergies Chine $\mathrm{n}^{\circ} 13$ - 2018 p. 131-142.

Laval, V., \& Bernicot, J. (2002). Tu es dans la lune : understanding idioms in French speaking children and adults. Pragmatics, 12 (4), 399-413.

Le Bel, E. (2006). Traduire la phraséologie: Réflexions méthodologiques et étude de cas. RæL 5, 2006, 57-70.

Lyster, R. (1994). The Effect of Functional-Analytic Teaching on Aspects of French Immersion Students' Sociolinguistic Competence. Applied Linguistics, Vol. 15, No. 3. 
Mede, E. et Dikilitaş, K. (2015). Teaching and Learning Sociolinguistic Competence: Teachers' Critical Perceptions. Participatory Educational Research (PER), 2(3);14-31, 1 December, 2015.

Mehdadi, Y. et Mokaddem, K. (2015). L'apport de la bande dessinée dans le développement de la compétence scripturale chez les apprenants de FLE. Cas de la deuxième année secondaire. Synergies Algérie n 22 - 2015 p. 131-141.

Mizne, C. A. (1997). "Teaching Sociolinguistic Competence in the ESL Classroom" Senior Thesis Projects, 1993-2002. https://trace.tennessee.edu/cgi/viewcontent.cgi?referer= https://www.google.com/\&httpsredir $=1 \&$ article $=1019 \&$ context=utk interstp2

Nikolaou, G. (2009). L'éducation interculturelle : un processus de compréhension et de communication dans le contexte scolaire.

Publibook, https://www.researchgate.net/publication/232296080_N ikolaou_G_L'education_interculturelle_un_processus_d e_comprehension_et_de_communication_dans_le_cont exte scolaire/

Nussbaum, L. et Unamuno, V. (2006). La compétence sociolinguistique, pour quoi faire? Dans Lorenza Mondada \& Simona Pekarek Doehler (Eds). La notion de compétence: études critiques. Bulletin suisse de linguistique appliquée, $\mathrm{N}^{\circ} 84,2006$, III.

Palloff, R. M., \& Pratt, K. (2005). Collaborating online: Learning together in community. San Francisco, CA: JosseyBass.

Papcov, I. (2012). La composante culturelle des expressions idiomatiques. LA FRANCOPOLYPHONIE 7, vol. I.

Paszenda, J. (1998). The Dog, Pig and Other Animals in Phraseological Units Depicting Human Unhappiness in English, Polish and German. Topics in Phraseology, Vol. 1. Katowice.

Petitpas, T. 2010. «Enseigner la variation lexicale en classe de FLE ». The French Review, 83(4), p. 800-818. 
Pulido, L., Iralde, L. \& Weil-Barais, A. (2010). Les expressions idiomatiques à l'école maternelle. Bulletin de Psychologie, 63, 469-480.

Qin, Z., Johnson, D.W., Johnson, R.T. (1995). « Cooperation Versus Competitive Efforts and Problem solving ». Review of Educational Research. Vol. 65 no 2.

Ritchie, M. (2011). Developing sociolinguistic competence through intercultural online exchange. In S. Thouësny \& L. Bradley (Eds.), Second language teaching and learning with technology: views of emergent researchers (pp. 123-141). Dublin: Research-publishing.net. Received from https://files.eric.ed.gov/fulltext/ED574607.pdf

Sioridze, M. et Abashidze, N. (2015). L'enseignement des Expressions Idiomatiques du FLE aux Apprenants Géorgiens. International Journal of Multidisciplinary Thought, 05(03):73-82.

Sioridze, M. et Surguladze, N. (2017). Les difficultés de compréhension de l'aspect culturel des expressions idiomatiques chez les apprenants de FLE. International Journal of Multidisciplinary Thought, CD-ROM. ISSN: 2156-6992: 06(02):103-114 (2017).

Škopíková, S. (2018). Development of sociolinguistic competence at lower-secondary schools. Ph.D., Faculty of Education, Palacky University in Olomouc. https://theses.cz/id/dr9evm/DIPLOMOV_PRCE_S_KO PKOV 2018.pdf

Stoytcheva, M. (2018). Students' perceptions of online collaboration in a distance learning French language course. AIP Conference Proceedings 2048, 020030 (2018); https://doi.org/10.1063/1.5082048.

Sułkowska, M. (2003). Séquences figées : Étude lexicographique et contrastive Question d'équivalence. Maison d'édition d'Université de Silésie, Katowice.

(2013). De la phraséologie à la phraséodidactique. Études théoriques et pratiques. Katowice : Wydawnictwo Uniwersytetu Śląskiego (Maison d'édition de l'Université de Silésie). 
(2016). Phraséodidactique et phraséotraduction : quelques remarques sur les nouvelles disciplines de la phraséologie appliquée. DE Gruyter Mouton, volume 7, Issue 1.

Tamayo, R. R. (2017). La Phraséologie : Le Statut des Expressions Idiomatiques dans le Système Linguistique Français. Thèse de magistère, Faculté de Filología - UNED.

Teixeira, V. (2011). Atelier évaluation et CECR: un lien de succès pour évaluer en français langue étrangère. Actes du XII ${ }^{\text {ème }}$ colloque pédagogique de L'Alliance Française de São Paulo. Reçu de http://www.aliancafrancesa.com.br/colloque2/actes/Act e Atelier VANILDA TEIXEIRA.pdf.

Walckiers, M. et De Praetere, T. (2004). L'apprentissage collaboratif en ligne, huit avantages qui en font un must. Distances et savoirs. Volume $2-\mathrm{n}^{\circ} 1 / 2004$.

Zhang, Y. (2012). Pour une approche interculturelle de l'enseignement du français comme spécialité en milieu universitaire chinois. Linguistics. Université du Maine, 2012. French. <NNT : 2012LEMA3010>. <tel00793142> 